\title{
Gradhiva
}

GRADHIV

Revue d'anthropologie et d'histoire des arts

29 | 2019

Estrangemental

\section{Manhattan Transfer. Benjamin Lee Whorf diffracté par Philip K. Dick}

Manhattan Transfer. Benjamin Lee Whorf diffracted by Philip K. Dick

\section{Pierre Déléage}

\section{(2) OpenEdition \\ Journals}

Édition électronique

URL : http://journals.openedition.org/gradhiva/4197

DOI : 10.4000/gradhiva.4197

ISSN : 1760-849X

Éditeur

Musée du quai Branly Jacques Chirac

Édition imprimée

Date de publication : 29 mai 2019

Pagination : 222-249

ISBN : 978-2-35744-112-5

ISSN : 0764-8928

\section{Référence électronique}

Pierre Déléage, « Manhattan Transfer. Benjamin Lee Whorf diffracté par Philip K. Dick », Gradhiva [En ligne], 29 | 2019, mis en ligne le 31 mars 2021, consulté le 02 avril 2021. URL : http://

journals.openedition.org/gradhiva/4197; DOI : https://doi.org/10.4000/gradhiva.4197

(c) musée du quai Branly 
$\frac{1}{4}$
0
0
0

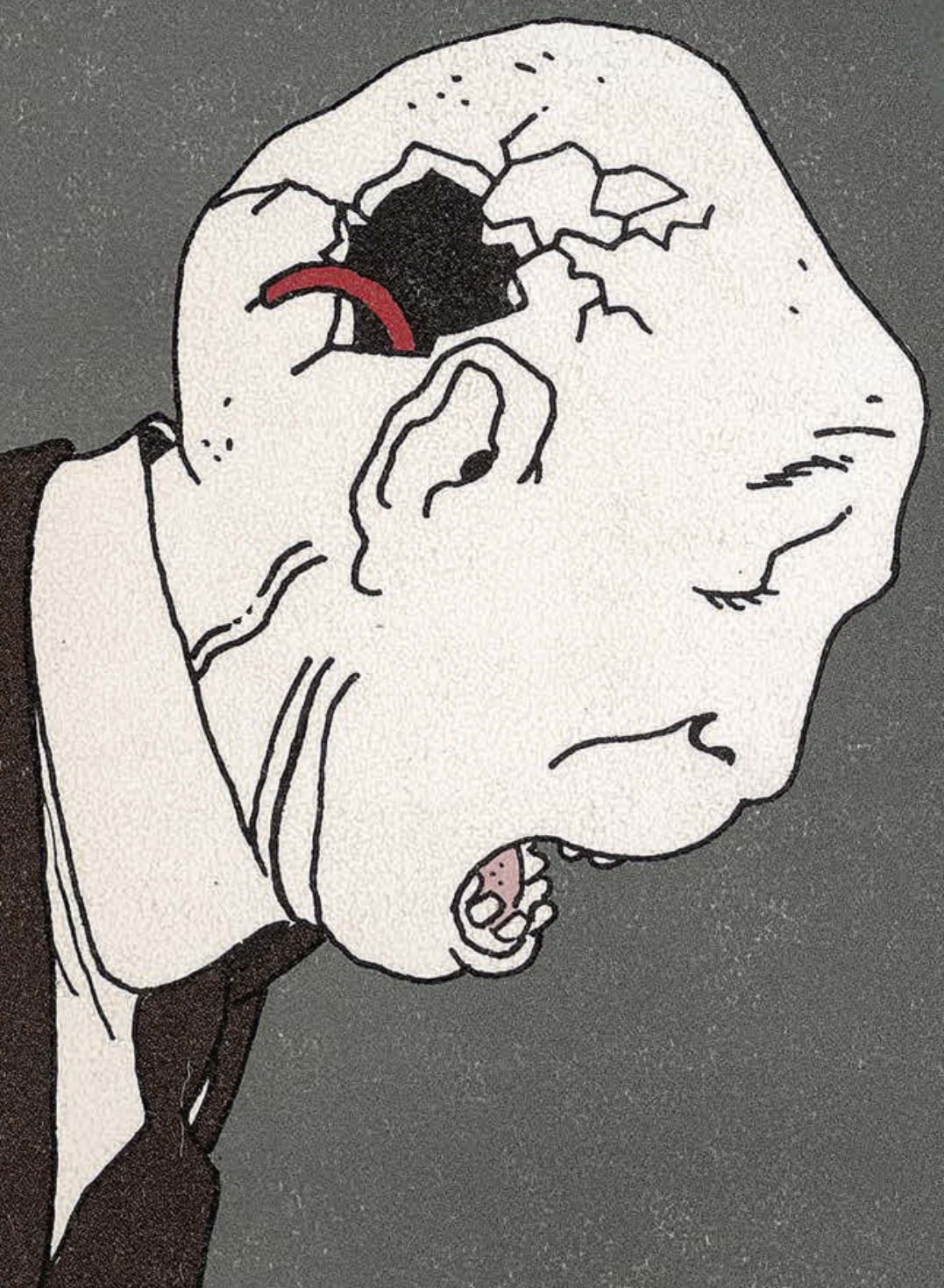

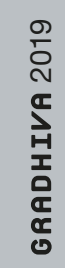




\section{Manhattan Transfer}

Benjamin Lee Whorf diffracté par Philip K. Dick

par Pierre Déléage

On dit des anthropologues qu'ils sont par vocation des spécialistes du relativisme. En étudiant les langues, les mythes, les rituels de sociétés au mode de vie le plus éloigné possible du leur, ils semblent souvent tourmentés par la recherche d'une pensée véritablement autre. Parfois, cette quête les fait trébucher et imaginer de toutes pièces une métaphysique nouvelle, étrange, mystérieuse, qu'ils imputent à une petite société méconnue. C'est le cas de l'ingénieur Benjamin Lee Whorf (1897-1941). En marge de l'école boasienne, il inventa une philosophie de l'événement et la présenta comme immanente à la langue et à la culture des Hopi d'Arizona. L'anthropologue se faisait ainsi écrivain de science-fiction. Une confrontation de ses travaux avec les ressorts de l'œuvre de Philip K. Dick, qui, lui aussi, n'a cessé d'imaginer des métaphysiques stupéfiantes et d'en dérouler les conséquences, permet de mettre au jour certaines des conditions fantasmatiques de l'anthropologie et d'explorer les rapports parfois subtils qu'entretiennent la science et la fiction. 
1. Toutes les citations ont été traduites par l'auteur.
Dans le deuxième quart du $x x^{e}$ siècle, l'ingénieur Benjamin Lee Whorf, plutôt que d'essayer de décrire le monde et la pensée de la société qu'il se proposait d'étudier, finit par les inventer. À la recherche d'une pensée autre, il projeta sur la réalité ethnographique des Hopi d'Arizona une forme de pensée dérivée pour l'essentiel de son imaginaire et de problèmes propres à son milieu intellectuel d'origine. En marge de l'école boasienne, il élabora ainsi une pensée de l'événement qu'il voulut immanente à la langue des Hopi. Comme les écrivains de science-fiction, il rejeta les projets réaliste et naturaliste pour créer un monde et un mode de pensée stupéfiants. À la différence de ces écrivains, il n'assuma jamais le caractère fictionnel de ses travaux.

Auteur dont l'œuvre a atteint un des sommets de la science-fiction du $x x^{e}$ siècle, Philip K. Dick n'a cessé d'élaborer des dispositifs où non seulement un mode de pensée délirant se substituait à un mode de pensée ordinaire, mais où ces modes de pensée transitaient, par l'effet d'une commutation ou d'un escamotage, d'un personnage à un autre, d'une subjectivité à une autre. Ses romans et nouvelles concernent au premier chef les anthropologues en quête de pensée autre, en particulier ceux qui ne furent pas très regardants quant à la réalité des sociétés qu'ils décrivirent. Philip K. Dick savait en effet très bien que la distinction entre réalités subjective et objective était facile à estomper en littérature et il fit de ce simple trucage ontologique la principale cheville de son outillage créatif.

Le texte qui suit s'empare des ressorts métaphysiques constitutifs de l'œuvre de Philip K. Dick pour les faire résonner, au moyen d'un montage parallèle, avec les fabulations théoriques de Benjamin Lee Whorf. L'objectif de ce dispositif croisé est double: d'une part donner à voir clairement et simplement l'équivalence, au cœur d'une œuvre exemplaire, entre anthropologie et science-fiction; d'autre part faire ressentir, par un réseau très dense d'associations et de correspondances alternées, la sensibilité théorique constitutive de cette équivalence.

1925. Hartford, Connecticut. Benjamin Lee Whorf, ingénieur chimiste de 29 ans, achève son premier roman, The Ruler of the Universe, par la description du long cauchemar prophétique de John Landon, son alter ego traumatisé par les atrocités de la Première Guerre mondiale, qui voit à travers les nuages un aéronef larguer sur une ville une multitude de bombes de gaz. "La ville gémit comme un condamné entrevoyant son agonie future 1 " et la course effrénée aux armements, conduite par des scientifiques inconscients, précipite le jugement dernier, l'annihilation chimique d'une humanité abandonnée par Dieu (Rollins 1971). 1924. Dans un essai livré à l'American Mercury, Edward Sapir souligne «la relativité des formes de pensée» enracinée dans les interprétations incommensurables de la réalité issues de langues différentes; il ajoute que, malgré les œillères que nous avons héritées de nos langues et de nos modes de pensée figés, cette idée «n'est pas aussi difficile à comprendre que la théorie de la relativité physique d'Einstein » (Darnell 2001: 178). 


\section{2}

En français comme en anglais, on parle de «dix femmes» comme on parle de «dix jours». Pourtant si, dans le premier cas, il sera toujours possible d'observer objectivement et simultanément dix personnes à la fois, dans le second, ce n'est que par une abstraction conceptuelle que l'on parle de « dix jours ", tant il est vrai que nous ne pouvons en fait connaître qu'un seul jour, aujourd'hui. Cette abstraction est nommée «pluriel imaginaire» et elle s'oppose au pluriel réel des dix femmes. Dans la langue des Hopi, un peuple amérindien vivant au nord-est de l'Arizona et comptant quelque cinq mille locuteurs, il n'existe pas de pluriel imaginaire. Un énoncé associant un nombre cardinal ou tout simplement un pluriel à une entité abstraite, c'est-à-dire ne pouvant être décomptée qu'en faisant appel à l'imagination, ne peut être formulé en hopi. II n'existe donc pas d'expression telle que «dix jours». À la place on emploie une journée particulière déterminée par le biais d'un calcul approprié.

«Ils restèrent dix jours » devient «lls restèrent jusqu'au onzième jour » ou «lls partirent après le dixième jour». «Dix jours est un laps de temps supérieur à neuf jours » devient «Le dixième jour est postérieur au neuvième». (Whorf 2012 [1956]: 180)

La langue hopi, dans ce contexte grammatical, ne quantifie pas le temps, elle l'ordonne. Sa logique est ordinale et non cardinale. Plutôt que de mesurer le temps, elle appréhende les relations d'antériorité et de postérité entre deux événements ${ }^{2}$. De ce phénomène purement linguistique, Benjamin Lee Whorf déduisit que les Hopi envisageaient le temps d'une manière radicalement différente de la «nôtre».

\section{3}

Les univers fictionnels de Philip K. Dick regorgent de commutateurs. L'un des plus étonnants est la "chambre de ralentissement » de Glissement de temps sur Mars (1962). Le récit prend place en 1992 sur Mars. La planète rouge, aride, est couverte de canaux d'irrigation - observés autrefois par Giovanni Schiaparelli - bâtis par une civilisation vieille de cinq mille ans, dont il ne reste dorénavant plus que des vestiges. De nombreux colons, profitant de l'occasion d'échapper à une Terre surpeuplée, polluée et irradiée, s'y sont installés à partir des années 1970, découvrant, errant parmi les ruines archéologiques, une population indigène clairsemée, nomade, vivant exclusivement de la chasse et de la cueillette, les Bleeks. La première moitié du roman décrit le cours presque ordinaire d'une journée de la vie martienne. Elle ressemble par bien des aspects à celle des banlieues américaines des années 1950, avec ses querelles de voisinage, ses commérages, ses VRP, etc. Point d'orgue de cette banale journée où tous les personnages sont présentés, le suicide de Norbert Steiner, commerçant dépressif, dont le père était phocomèle et le fils schizophrène.

Le frêle garçon de 10 ans, Manfred, n'a jamais prononcé un mot. Gracile, il ne se déplace qu'en dansant sur la pointe des pieds. Son psychiatre, le Dr Milton Glaub, pense qu'il est peut-être doué de facultés précognitives, c'est-à-dire de prédiction de l'avenir. Il propose à Arnie Kott, le chef du syndicat
2. Voir aussi Whorf 1946 : 165 et $170 ;$ p. 179 il est noté qu'il existe une exception: l'expression en années de l'âge d'une personne suit une logique cardinale. L'absence de pluriel en hopi pour le mot «jour » et, partant son fonctionnement ordinal, est confirmé par Malotki 1983: 241 sq. 


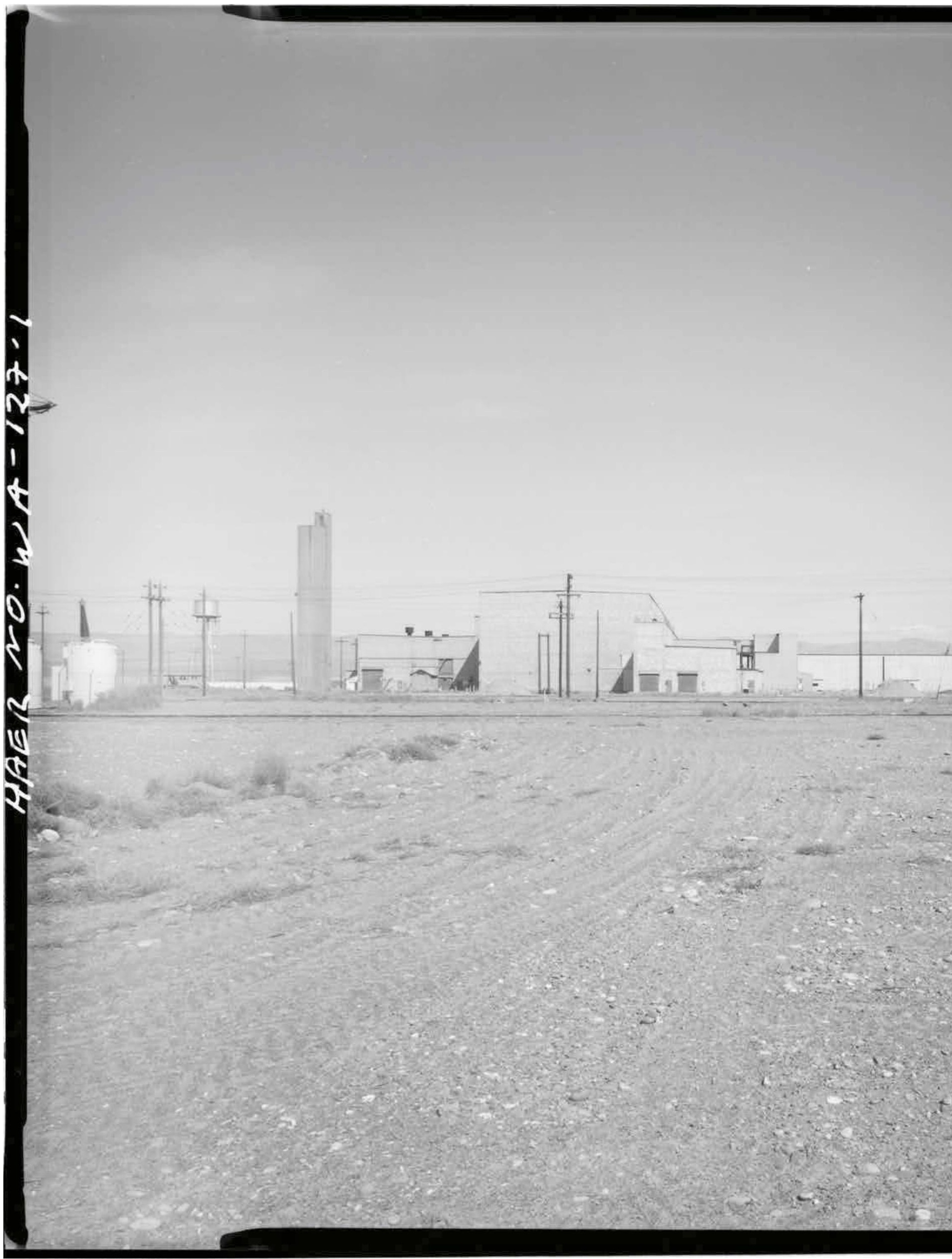




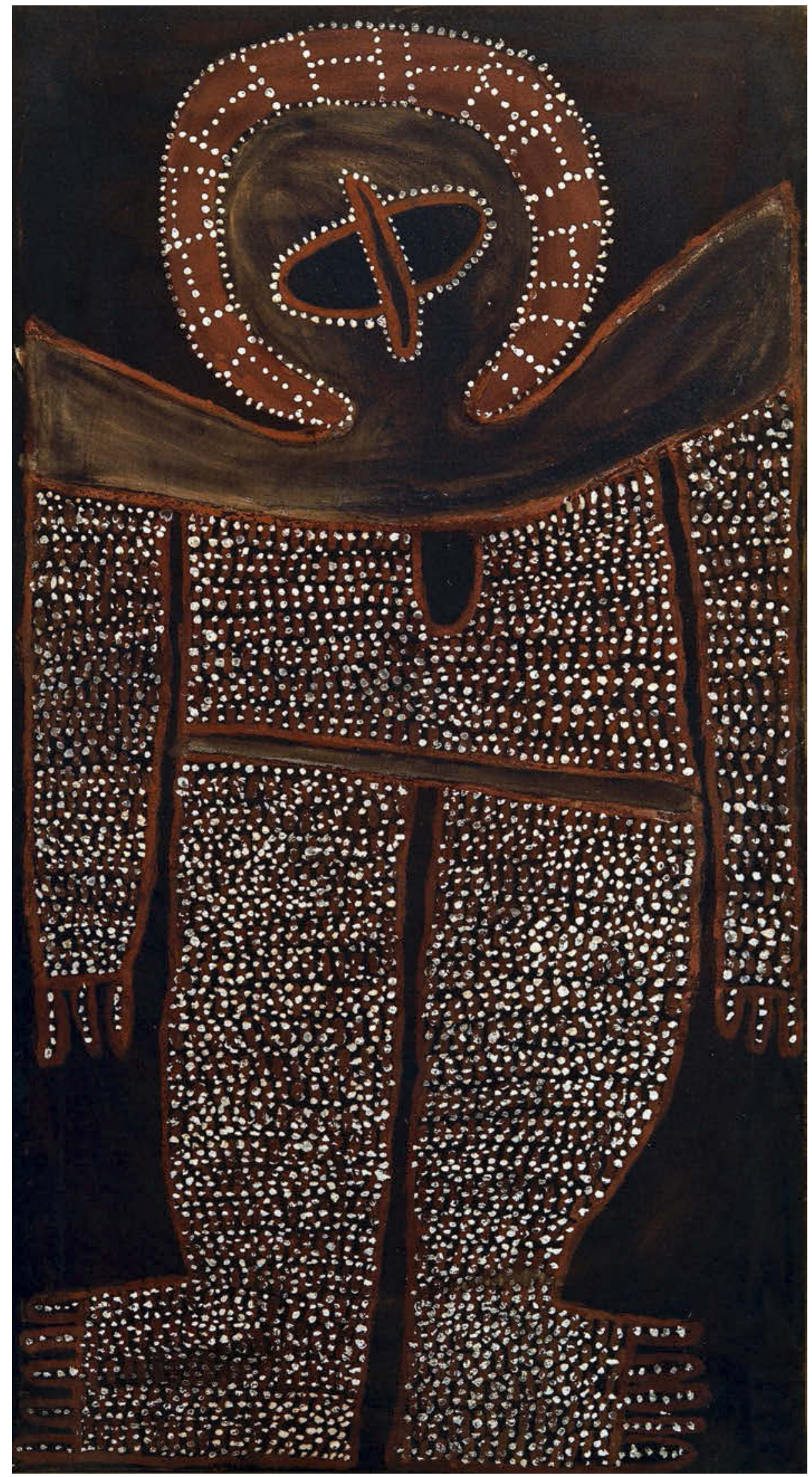


des plombiers, l'un des hommes les plus riches et les plus puissants d'une planète où l'eau est une ressource précieuse, de fabriquer une machine afin de pouvoir communiquer avec l'enfant et, partant, de tirer avantage de ses possibles visions du futur. S'appuyant sur l'analyse existentielle développée par l'école suisse de Burghölzi, le psychiatre assimile la schizophrénie à une profonde détérioration de la perception du temps qui, dans le roman, prend deux formes distinctes. L'écoulement du temps subjectif du schizophrène peut être d'une certaine manière interrompu: il ne perçoit plus le monde que sur le plan de l'éternité, sa conscience embrassant simultanément le futur, le passé et le présent (un «retour au mode de pensée primitif»). C'est Jack Bohlen, un réparateur de machines, qui souffre de cette forme de schizophrénie. Mais dans d'autres cas, ce peut être l'écoulement du temps propre à l'environnement, du temps «objectif», qui est accéléré, et alors le schizophrène ne voit plus que des images floues et n'entend plus que des voix suraiguës. Comparé à la vélocité de ce temps objectif, le temps subjectif de Manfred Steiner, sa durée intérieure, est ralenti, ce qui provoque l'effondrement de ses facultés de communication et sa coupure définitive d'avec le monde social.

L'idée du Dr Glaub est simple: pour restaurer le contact avec Manfred, il suffira de construire une chambre close dans laquelle des projecteurs feront défiler sur les murs des films au ralenti, tandis que des magnétophones, aux bandes réglées elles aussi au ralenti, diffuseront des paroles enregistrées. En adaptant l'image et le son au rythme de la temporalité subjective de l'enfant, il deviendra possible de lui apprendre à parler, en faisant coïncider images et sons, puis de communiquer avec lui. Il est calculé que le temps de Manfred est 4,22 fois plus lent que celui des humains non psychotiques, ce sera donc le facteur de ralentissement des machines audiovisuelles. Ce commutateur particulièrement inventif, destiné à faire le lien entre deux réalités hétérogènes, entre deux temporalités, échouera lamentablement.

\section{4}

1936. Quelque part dans Manhattan, Ernest Naquayouma, Hopi originaire du village de Toreva mais dorénavant résident à New York, rejoint Benjamin Lee Whorf pour lui enseigner sa langue maternelle (Whorf 1946 : 158). 1939. Franklin Roosevelt, président des États-Unis, reçoit le 10 octobre une lettre d'Albert Einstein l'informant de la possibilité imminente de déclencher une réaction nucléaire en chaîne avec de grandes quantités d'uranium, phénomène qui permettrait la confection de bombes d'un genre nouveau, d'une puissance difficile à imaginer (Einstein 1939). 1938. Alfred Korzybski, ingénieur chimiste, inaugure à Chicago le premier Institut de sémantique générale où, en s'affranchissant de la malédiction aristotélicienne du langage ordinaire, on pourra recouvrer la santé mentale (Schuchardt 1950). 1919. Benjamin Lee Whorf, fraîchement diplômé du Massachusetts Institute of Technology, est embauché par la Hartford Insurance Company pour inspecter des usines ou des maisons dévastées par des incendies ou des explosions et rédiger des rapports sur les circonstances qui en sont à l'origine (Carroll 2012: 4). 1941. Benjamin Lee Whorf, 44 ans, sur le point de mourir d'un cancer, devient vice-directeur de sa compagnie d'assurances (Ibid.: 21). 1929. Alfred North Whitehead fait paraître Procès et réalité, la troisième grande logique de l'événement (Deleuze 1988: 72).

\footnotetext{
double page précédente

fig. 1

Historic American Engineering Record, D-Reactor Complex, Area 100-D, Richland, Benton County, WA. Un des complexes construits durant le Manhattan Project, s.d. (documentation compilée après 1968). Washington D.C., Library of Congress, inv. HAER WASH, 3-RICH.V,1.

ci-contre

fig. 2

Alec Mingelmanganu,

Wandjina, vers 1980.

Pigments naturels sur toile,

$121 \times 66 \mathrm{~cm}$. Art Auction,

Sotheby's, Melbourne/28

juin 1999/akg-images.

Droits réservés.
} 


\section{ci-contre}

fig. 3

Jacques Tardi, illustration pour Le Bal des schizos de Philip K. Dick. Paris,

Champ libre, 1975

(C) Jacques Tardi.

Benjamin Lee Whorf, comme de nombreux savants et ingénieurs de son époque, avait reçu de plein fouet les révélations de la théorie de la relativité (Rollins 1980: 25-30; Heynick 1983). Même si le principe d'inertie de Galilée ou la gravitation de Newton avaient déjà peiné à s'accorder avec le sens commun, les propositions d'Einstein le violaient sans scrupule, avec constance et minutie: une vitesse de la lumière constante pour tous les observateurs, quelles que soient leurs vitesses relatives; un temps qui, perdant son universalité, se dilate et se contracte; un seul espace-temps recouvert d'une multitude de systèmes de référence inertiels; un nombre illimité de géométries non euclidiennes qui donnent une description également parfaite des configurations spatiales. La physique nouvelle mettait le philosophe dans la situation d'avoir à repenser les concepts obsolètes d'espace, de temps ou de matière hérités de la science newtonienne. Le langage mathématique, en s'affranchissant des contraintes de la langue, avait rendu possible un nouvel entendement des lois de l'univers. Cependant, cette nouvelle compréhension semblait adverse à toute formulation en langue ordinaire. Les idées de la théorie de la relativité se heurtaient frontalement au "sens commun" de l'Occident, "non parce que la nature elle-même les contredit, mais parce que la manière dont elles doivent être exprimées équivaut à parler un langage nouveau » (Whorf 2012 [1956]: 195).

De là il n'y avait qu'un pas pour concevoir la «pensée scientifique" traditionnelle, pré-relativiste, comme une simple «spécialisation du langage indo-européen de type occidental ». Si les concepts scientifiques formulables à l'aide des mathématiques étaient devenus inintelligibles lorsque formulés en langue «indo-européenne de type occidental », c'est que cette langue véhiculait avec elle un "sens commun", une vision du monde particulière. Et la science d'Einstein était «en train de découvrir qu'il y a quelque chose dans le cosmos qui n'est pas en accord avec les concepts que nous avons formés " dans le cadre des langues européennes (Ibid. : 197). La contre-intuitivité de la théorie de la relativité était donc pour Benjamin Lee Whorf la preuve des limites des langues "européennes", qui avaient réussi le tour de force de faire passer pour intuitifs les soubassements conceptuels de la science newtonienne. Partant, le groupe des langues "européennes" contenait implicitement une métaphysique particulière, différente de celle, plus vaste, rendue possible par le langage mathématique. Dès lors il n'y avait pas de raison pour que d'autres langues, non européennes, n'exprimassent pas une autre métaphysique. Benjamin Lee Whorf partit en quête d'une telle métaphysique autre.

Ainsi chaque langue «exprimait un champ de référence cosmique». Elles «cristallisaient en elles-mêmes les postulats de base d'une philosophie informulée dans lesquels était contenue la pensée d'un peuple, d'une culture, d'une civilisation, voire d'une ère» (Ibid. : 78). Whorf dut donc dans un premier temps définir la métaphysique issue des langues "européennes", métaphysique constitutive du matérialisme newtonien classique. Pour lui, cette métaphysique portait essentiellement sur ce qu'on appelle "les choses" dotées de modalités d'existence extensionnelles mais informelles, désignées sous le nom de "substance » ou «matière». Elle analysait le réel au travers d'une formule binôme, typique de la logique du pluriel des noms de masse, où une forme spatiale venait s'appliquer à une substance informelle 


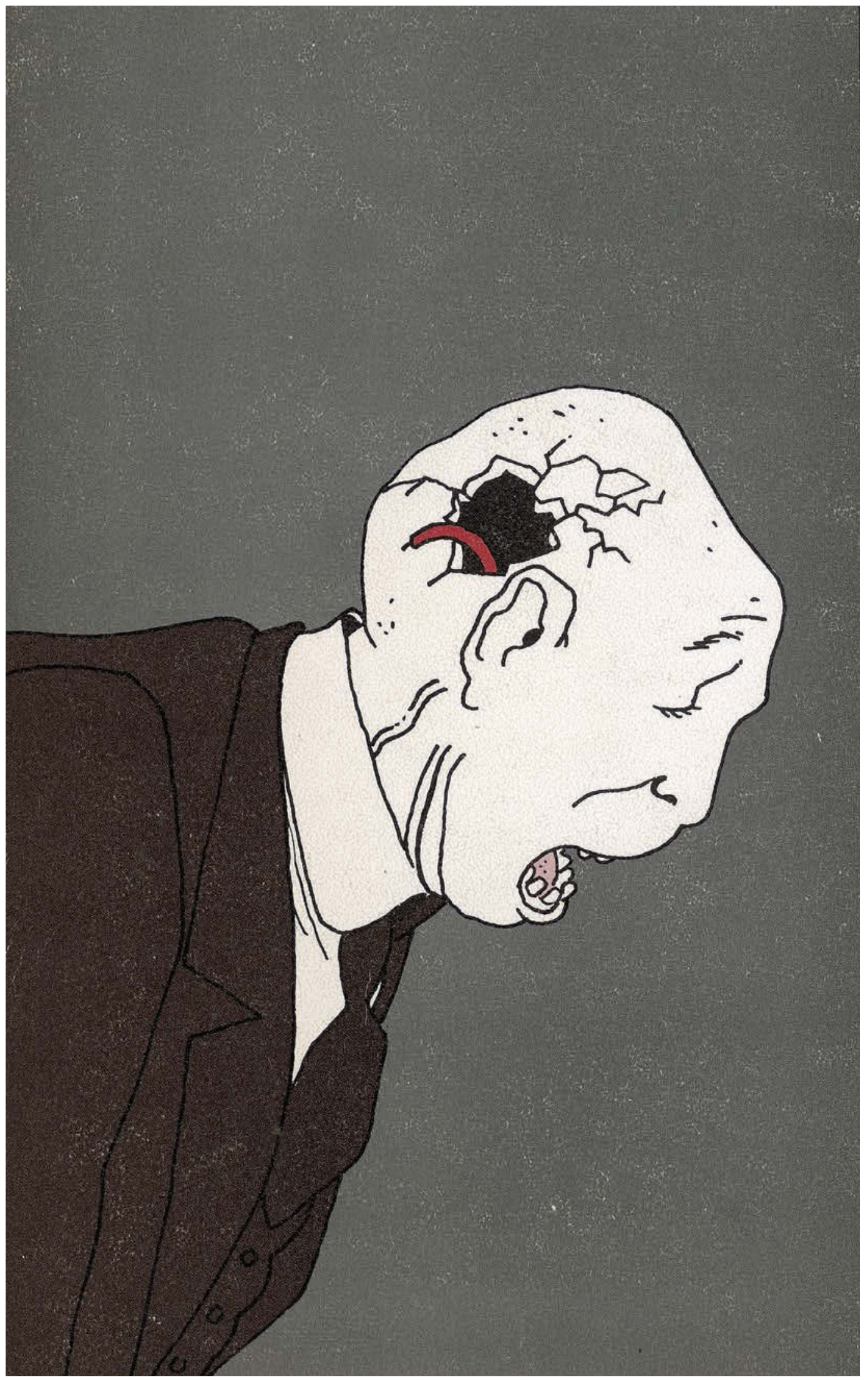


(«des morceaux de viande» et non «des viandes»). Les étants non spatiaux étaient dès lors spatialisés par l'imagination qui leur attribuait une forme s'inscrivant dans un continuum - à l'image du monde matériel (Whorf 2012 [1956] : 189). Cet hylémorphisme engendrait, à l'aide d'une structure dichotomique et d'une extension métaphorique imaginaire, d'une part un espace atemporel, homogène, immédiat, tridimensionnel, infini et statique, et d'autre part un temps objectivé, unidimensionnel, éternellement et uniformément mouvant, scandé par une division ternaire - passé, présent, futur (Ibid. 75-76). C'était là la métaphysique commune que nous avions héritée de la grammaire des langues que nous parlons et qui s'était subrepticement glissée dans les premières formulations de la science occidentale.

À partir d'un tel postulat établissant l'homologie entre les structures des langues européennes et les concepts de base de la science newtonienne, Benjamin Lee Whorf souhaita entreprendre l'étude d'une langue exotique, d'une langue qui lui révélerait une métaphysique nouvelle, une métaphysique aussi nouvelle que celle qu'il peinait à déduire de la théorie de la relativité. II choisit pour cette étude la langue hopi.

La langue et la culture hopi recèlent une métaphysique, au même titre que notre conception prétendument naïve du temps et de l'espace, ou que la théorie de la relativité; il s'agit cependant d'une métaphysique qui diffère de celle-ci. (Ibid. : 74)

En effet, Benjamin Lee Whorf considérait la linguistique comme un verre grossissant à travers lequel, quand la mise au point était correcte, se profileraient un jour les vraies formes de beaucoup de ces forces qui jusqu'ici n'avaient été pour l'humanité qu'un no man's land impénétrable, domaine d'une activité mentale invisible et désincarnée. II trouverait dans la métaphysique hopi (métaphysique entièrement comprise dans les structures explicites mais surtout implicites de leur langue) les moyens d'une nouvelle synthèse transcendantale de l'expérience et de l'intuition des choses, relativisant les catégories de la pensée occidentale pour atteindre un entendement plus vaste, un entendement de nature finalement mystique (Ibid. : 75). Apprendre la langue hopi, c'était immédiatement accéder à une nouvelle pensée et à un nouveau monde.

\section{6}

Des commutateurs de toutes natures sont donc au fondement de ce que Philip K. Dick nommait son «plurivers» (Briggs et Scott Apel 1992: 90). Toutefois limiter à l'existence d'une infinité de réalités parallèles la cosmologie sous-jacente aux textes de Dick, en particulier ceux des années 1950 et 1960 , ne serait pas rendre justice à leur complexité. Ce serait oublier que la cosmologie dickienne est avant tout narrative, c'est-à-dire qu'elle prend moins forme dans l'argumentation et la typologie que dans les ressorts le plus souvent psychologiques d'une intrigue. Ainsi les grands textes cosmologiques de Dick commencent toujours, plutôt que par un doute hyperbolique, par un simple sentiment de contre-factualité, sentiment qui peut, selon les récits, recouvrir une large variété de nuances. 
Quelle autre réalité se cache sous une imitation de la réalité ? La reconstitution de la ville peut-elle devenir la ville? Ce sont là deux problèmes métaphysiques qui dominent toute l'œuvre dickienne. Le premier est le plus classique. C'est par exemple l'une des lignes narratives de Glissement de temps sur Mars. Le réparateur Jack Bohlen connaît à intervalles réguliers des crises de «schizophrénie» pendant lesquelles il voit une réalité parallèle. Les gens lui apparaissent alors comme des cadavres mécaniques.

II vit le psychiatre dans sa réalité absolue: une chose froide composée de fils métalliques et de boutons, totalement inhumaine, sans la moindre chair. La carapace charnue se décomposa, devint transparente, et Jack Bohlen aperçut les mécanismes installés à l'intérieur. Cependant il ne laissa rien paraître de son terrible discernement; il continua de bercer tranquillement son verre, d'écouter la conversation en hochant la tête de temps en temps. Le Dr. Glaub ne s'aperçut de rien. (Dick 2014a: 140)

Et lorsque la crise est finie, il demeure hanté par cette question: et si la réalité qui lui apparaissait durant ces épisodes n'était pas une hallucination, si elle était la vraie réalité? Dick maintient le doute: il pourrait virtuellement y avoir deux réalités parallèles.

Les fictions de Philip K. Dick se font plus intéressantes encore Iorsque, au-delà de cette omniprésente ambiance d'incertitude ontologique, l'auteur introduit le principe des contaminations de réalités. C'est probablement son innovation majeure, sa signature. Comme souvent dans la sciencefiction, il s'agit de jouer avec une certaine forme de littéralité concernant la communication et l'empathie. Si la communication est avant tout la reconstruction du point de vue d'autrui, elle est fondée sur des expressions courantes telles que «se mettre à la place de l'autre» ou «prendre son point de vue sur les choses ", expressions qui doivent bien évidemment être entendues au sens figuré. Prises à la lettre (Lehman 2001), ces expressions sont au fondement de l'ontologie perspectiviste de Dick, ontologie qui ne peut être comprise qu'en relation avec son art littéraire. Car Dick emploie la plupart du temps une technique, par ailleurs assez courante, de narration multifocale: il n'existe dans ses textes aucun narrateur omniscient et donc aucune réalité de référence. Le récit est raconté par l'intermédiaire d'une série de focalisations internes partiales et partielles, par l'alternance des points de vue propres à chacun des personnages; il obéit à « une structure entrelaçant une multiplicité de systèmes perceptifs monadiques dans laquelle la "réalité" est toujours subjective et donc variable d'un personnage à l'autre " (Dick 1996a: 77-78). La réalité, telle qu'elle émerge de la composition des fictions dickiennes, apparaît en somme comme un interfaçage de réalités subjectives.

On en trouve un précoce exemple dans Le Monde qu'elle voulait (1952). Le narrateur, Larry Brewster, rencontre dans un bar de jazz la jeune Allison Holmes qui l'entraîne à sa suite pour une virée festive et philosophique dans la nuit new-yorkaise. Elle lui fait progressivement comprendre qu'ils sont tous deux dans son monde à elle, un monde qui obéit entièrement à ses désirs. Et, peu à peu, le narrateur doit admettre que tout se passe autour d'eux «comme elle le veut». Larry Brewster comprend donc qu'il est «pris» dans le monde subjectif d'Allison Holmes, monde qui n'en a pas moins une réalité tangible. Elle lui explique que lui aussi a certainement son monde à lui, 


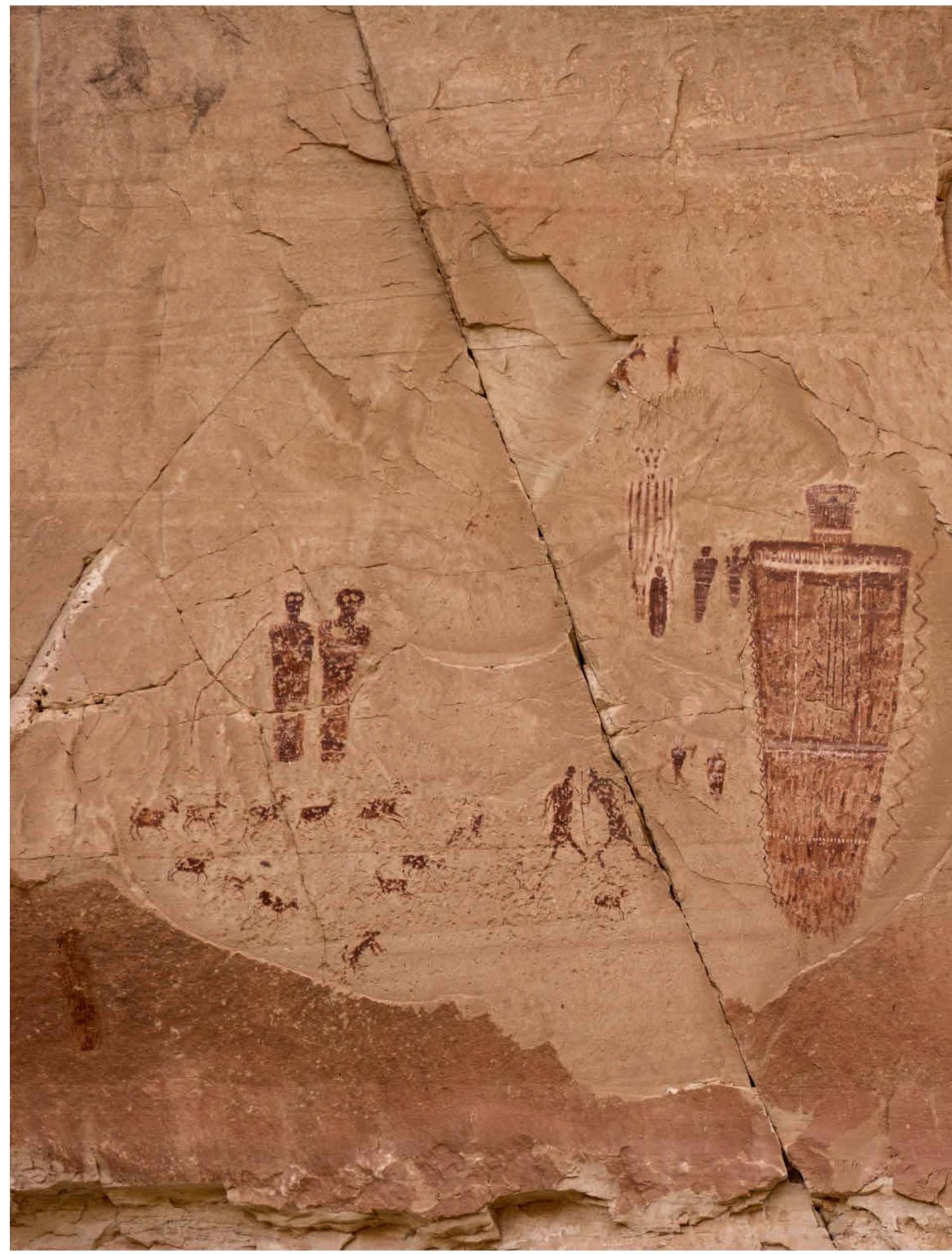




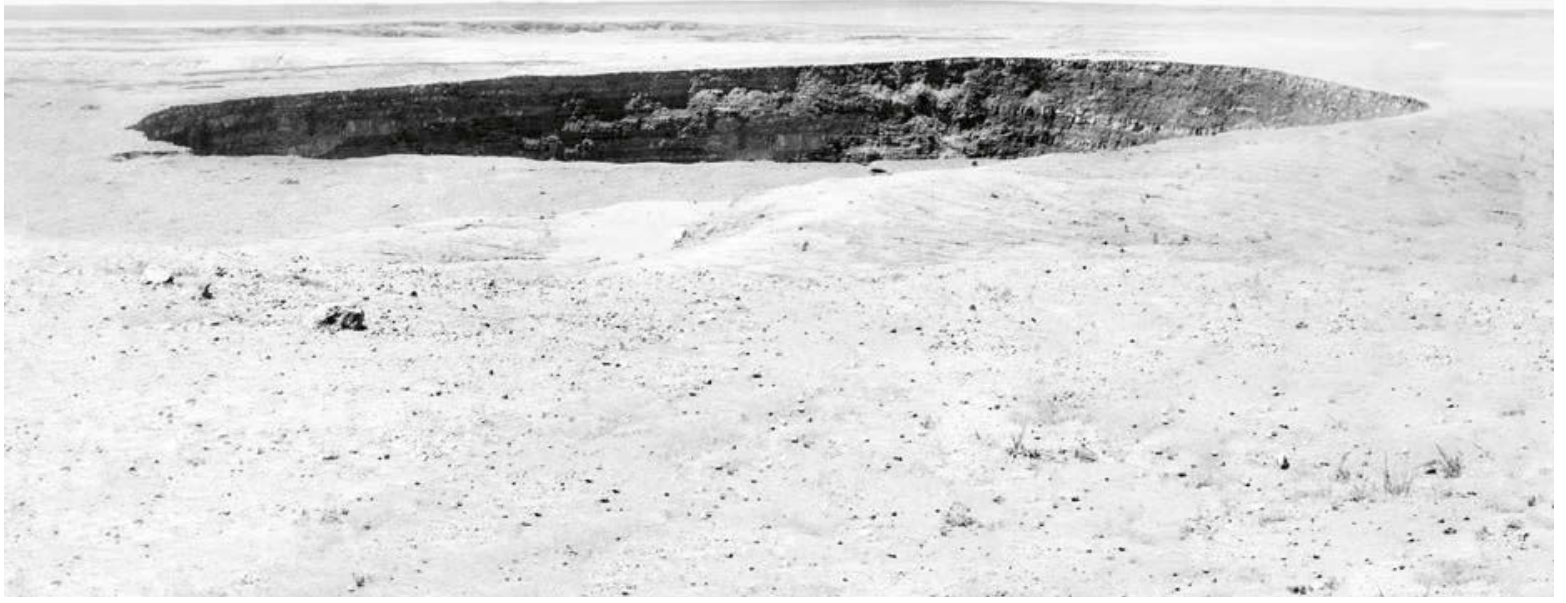


mais que dans ce monde-là il n'est que l'objet virtuel de son désir à elle. La focalisation de la nouvelle, entièrement interne à Larry Brewster, est ainsi un essai de réponse à la question: que pense un être imaginaire, fantasmé? Le récit s'achève par un retournement classique: épuisé par cette virée nocturne, Larry Brewster fait brusquement disparaître Allison Holmes. Ils étaient en fait dans son monde subjectif à lui et c'est elle qui n'était qu'un fantasme. Dans ce récit profondément dickien, le réel objectif disparaît au profit de deux réalités subjectives enchâssées, l'une illusoire, l'autre réelle ${ }^{3}$.

\section{7}

1922. Henri Bergson, lors de la discussion à la Société française de philosophie en date du 6 avril, essaie de régler ses comptes avec Albert Einstein qu'il rencontre pour la première et dernière fois. II plaide pour une conceptualisation philosophique de la simultanéité; Einstein lui oppose, sans plus y penser, une simple fin de non-recevoir (Bergson 2009: 391-398). 1936. Benjamin Lee Whorf, 39 ans, étudie la linguistique à l'université de Yale sous la direction d'Edward Sapir, qui dit de lui à Alfred Kroeber:

C'est un homme diablement doué, essentiellement autodidacte, doté d'un soupçon de génie. Il est parfois enclin à s'écarter du problème central pour s'égarer dans des spéculations marginales passionnées mais ce n'est là que la preuve de l'originalité d'un esprit aventurier. Il est le meilleur spécialiste de langue amérindienne dont nous disposons en ce moment. (Darnell 2001: 182)

1963. Il est douze minutes avant minuit sur l'horloge du Bulletin of Atomic Scientists et dans la deuxième livraison de l'année, le physicien Robert Livingstone s'étonne que les Hopi pensent naturellement en termes d'espace-temps, alors qu'il a fallu à Einstein sept années d'intense labeur pour remettre en question le concept de simultanéité absolue (Livingstone 1963: 16). 1925. Dans son roman The Ruler of the Universe, puis dans un essai sobrement intitulé Pourquoi j'ai rejeté la théorie de l'évolution, Benjamin Lee Whorf intente le procès d'une science matérialiste, inhumaine, suicidaire et maudite (Rollins 1980: 19).

\section{8}

La linguistique métaphysique de Benjamin Lee Whorf était une entreprise héroïque. Car il la savait condamnée, lorsque formulée en langue anglaise, à demeurer «inappropriée », à n'être qu'une «approximation inadéquate» (Whorf 2012 [1956]: 75). II avança malgré tout. II découvrit que la langue hopi induisait nécessairement une analyse globale des situations considérées sous l'angle des événements.

Objectivement, et seulement en tant qu'expériences physiques perceptibles, les «événements » s'expriment pour l'essentiel sous le rapport de la configuration externe, de la couleur, du mouvement et d'autres
3. C'est aussi un des rares récits de Dick où le narrateur est non fiable; en général c'est plutôt le monde lui-même qui n'est pas fiable pour le narrateur. double page précédente

fig. 4

Utah, Canyonlands National Park, Horseshoe Canyon Unit, The Great Gallery, Barrier Canyon style rock art. Photo John Elk III/Getty Images.

\section{ci-contre}

fig. 5

David de Beyter, Crater of the moon - II, 2016. Avec l'aimable autorisation de l'artiste. 
4. Ce qui est évidemment linguistiquement faux, voir Malotki 1983.

5. Pour une démonstration convaincante du contraire, voir Malotki 1983: 13-238.

6. Sur les tenseurs, voir l'étrange article de Lee 1991. éléments de perception. Subjectivement, qu'ils soient de nature physique ou non, les événements sont considérés comme l'expression d'invisibles facteurs d'intensité dont dépendent leur stabilité et leur continuité, ou leur fugacité et leur propension. (Ibid: 189-190)

Cette métaphysique de l'événement rendait soit très difficile, soit impossible à exprimer la notion, propre à la science newtonienne et aux langues européennes, de simultanéité absolue.

La métaphysique hopi ne soulève pas la question de savoir si les choses se trouvant dans un village éloigné existent au même instant du présent que celles situées dans le village où l'on se trouve. Elle affirme que tout "événement» survenant dans un village distant ne peut être comparé à un événement du village de l'observateur que par un intervalle de magnitude comprenant des formes à la fois spatiales et temporelles en lui. (Whorf 2012 [1956]: 80)

On comprendra dès lors pourquoi «la langue hopi ne possède pas de mot qui soit l'équivalent exact de notre temps» (Ibid. : 80), sans compter que «pas plus dans les verbes que dans les autres structures linguistiques hopi, il n'existe de possibilité d'expression pour un temps objectivé ${ }^{4}$ " (Ibid. : 186). Le temps hopi est en effet un processus de manifestation, un devenir toujours sur le point d'être ébauché, à jamais en train de ne pas vraiment finir. II s'exprime fondamentalement dans des modalités telles que l'expectatif ou I'inchoatif (Ibid. : 76-78). II est rétif à toute forme de métaphorisation, et en particulier d'objectivation spatialisée ${ }^{5}$. En lieu et place de ces transpositions mesurées, la langue hopi emploie des "tenseurs ${ }^{6}$ ", des particules qui «dépassent presque notre propre faculté d'appréhension» et qui qualifient l'intensité, la tendance, la durée et l'enchaînement logique des événements (Ibid. : 188-189; Whorf 1946: 179-180). En somme:

On peut considérer le hopi comme une langue intemporelle. II connaît un temps psychologique, qui ressemble beaucoup à la durée de Bergson, mais qui n'a rien à voir avec le temps mathématique $t$ utilisé par nos physiciens. Le temps hopi varie avec chaque observateur, il est incompatible avec la notion de simultanéité et il est adimensionnel; en d'autres termes on ne peut lui attribuer un nombre supérieur à un. (Whorf 2012 [1956]: 277)

La métaphysique hopi était donc fondée sur de purs événements, simples points s'inscrivant dans l'espace-temps («l'élément temporel ne peut être séparé des éléments spatiaux»).

En hopi, «éclair, vague, flamme, météore, bouffée de fumée, pulsation » sont des verbes - des événements d'une durée nécessairement brève ne peuvent être rendus que par des verbes. «Nuage » et «orage» sont à la limite inférieure de la durée pour des noms. Car la langue hopi, voyez-vous, procède en fait à une classification des événements fondée sur la notion de durée, ce qui est étranger à nos modes de pensée. (Ibid. : 276) 
À cette métaphysique linguistique de l'événement, Benjamin Lee Whorf associait un éthos culturel proprement hopi qu'il caractérisait comme une « attitude orante» (Ibid. : 79), une prédisposition au cérémonialisme le plus pointilleux. Et même si tout ce qu'il savait des Hopi se limitait à leur langue et à quelques lectures (il ne fit qu'un très bref séjour au village de Mishongnovi en 1938 à l'occasion d'un déplacement professionnel), il eut l'intuition que leur comportement se traduisait par une imprégnation du mental dans le cosmos, autrement dit par une préhension efficace de la perception et de la volonté, et par une douce obsession pour les préparatifs répétitifs et les préliminaires en général, une focalisation intense et systématique à la fois sur «l'annonce et sur l'attente d'événements qui se produiraient bien plus tard » (lbid. : 190-191).

\section{9}

Philip K. Dick a théorisé le perspectivisme immanent à son œuvre à l'aide de la distinction héraclitéenne entre idios kosmos et koinos kosmos, entre le monde privé des rêves et le monde partagé de l'état de veille (Dick 1996b, 1996c, 1996d, 1998; Platt 2015: 278). Ludwig Binswanger, la source de Dick, assimilait ces concepts à la distinction heideggérienne entre eigenwelt, le monde qui nous est propre, exclusivement, et mitwelt, le «monde avec ", le monde de l'ajustement et de l'adaptation aux relations sociales, délaissant, et c'est significatif pour la démarche dickienne, l'umwelt, le «monde alentour », le référent environnemental (May et al. 1958: 61-65).

Prolongement naturel de sa technique littéraire de narration multifocale, ces distinctions permettaient à Dick de prendre appui sur deux positionnements ontologiques. D'abord l'évacuation du troisième terme induisait l'absence de réalité référentielle ultime, objective, à partir de laquelle évaluer les réels subjectifs. Ensuite, si les subjectivités formaient le seul et unique kosmos possible, s'il n'y avait que des réels subjectifs, ceux-ci pouvaient être partagés (koinos) ou non (idios). Dick ne s'intéressait donc pas tant à la différence entre le réel et l'irréel qu'à la différence entre le réel subjectif (le monde subjectif d'Allison Holmes) et l'empiétement de réels subjectifs (le monde subjectif de Larry Brewster contaminé par celui d'Allison Holmes). Dans cette situation, le réel en tant que tel n'avait plus vraiment d'importance: le subjectif était l'unique réel et être pris dans le monde d'un autre, c'était littéralement être pris dans la «subjectivité faite monde» d'un autre. En d'autres termes le monde (du narrateur) était toujours susceptible d'être le monde d'un autre. Et si le monde d'autrui était le fruit de ses désirs fantasques, de ses idées farfelues, de ses cauchemars, ou même de sa folie, alors on se retrouvait d'emblée dans une fiction typiquement dickienne. Le substrat ontologique profond de l'œuvre de Dick est donc moins l'existence de réalités parallèles que celle de mondes privés pluriels.

Philip K. Dick admettait avoir trouvé cette idée dans un roman de Fredric Brown, L'Univers en folie (1949). Lorsque la fusée que l'on voulait envoyer sur la Lune tombe accidentellement sur la luxueuse villa où il était invité à dîner, Keith Winton, rédacteur en chef d'une revue de science-fiction populaire, est projeté dans un univers parallèle délirant qu'il découvre petit à petit. Situation classique de science-fiction. Keith Winton finit toutefois 


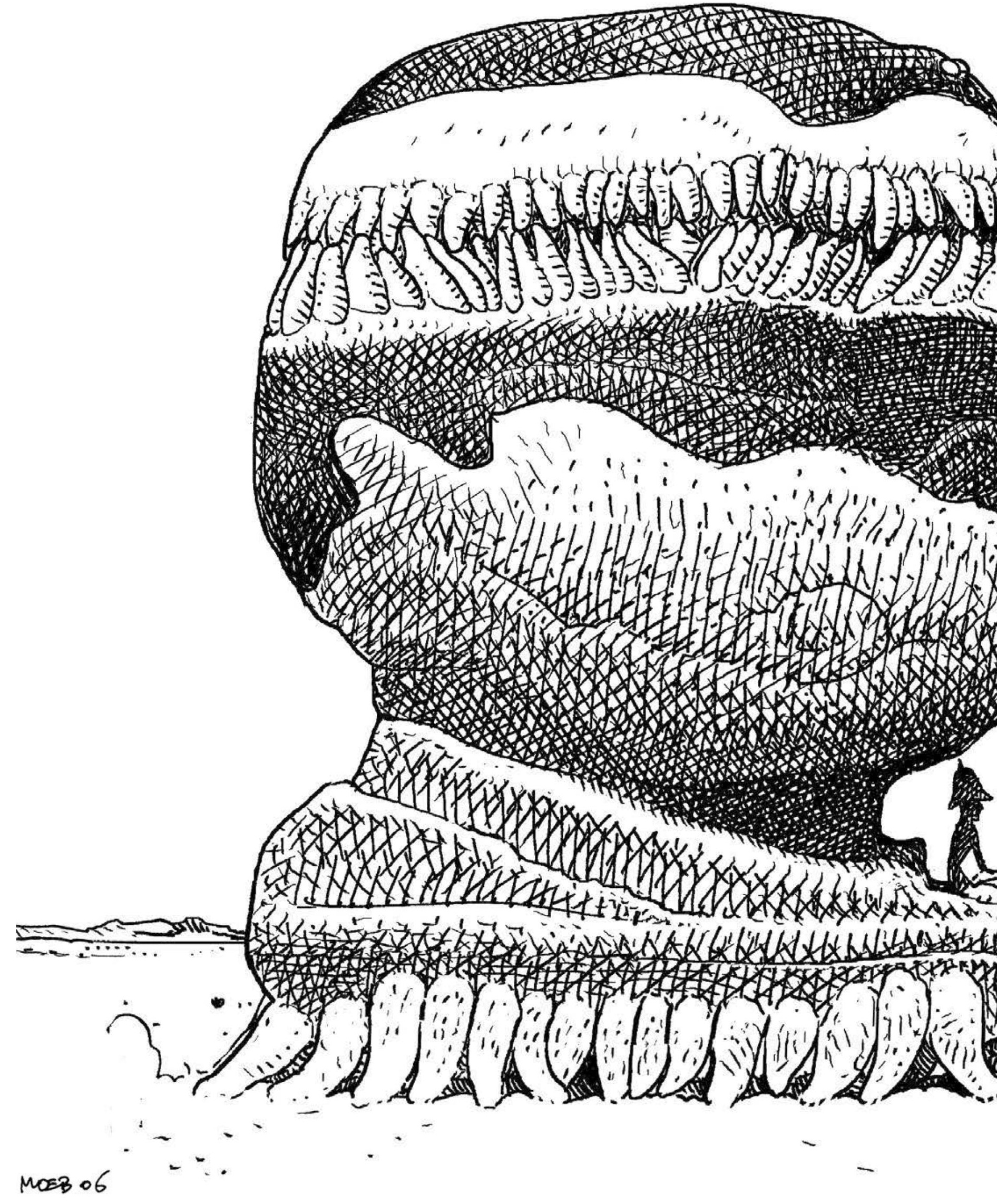



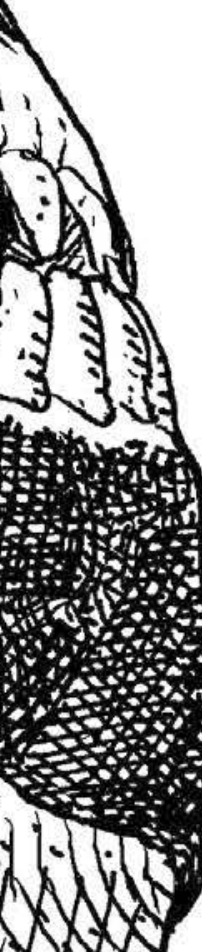

)

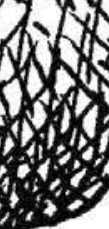

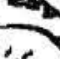

$\because 3$

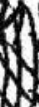

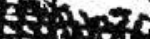

S 1304

.

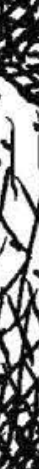


7. Indians at Work 11-3, 1943 : 27 ; Chicago Daily Tribune, 23 février 1950, cahier $4: 6$. par comprendre que le monde dans lequel il est plongé n'est autre que l'univers fantasmé d'un des lecteurs de sa revue: il se retrouve ainsi prisonnier du monde subjectif idéalisé d'un autre. Dick va développer ce double thème du monde subjectif devenu réalité et de la contamination de cette réalité dès L'CEil dans le ciel (1955), six ans après la parution du roman de Brown. Lors d'une visite du bévatron, l'accélérateur de particules de Berkeley, Bill Hamilton et un groupe de touristes, parmi lesquels Arthur Silvester, un ancien soldat très religieux, sont victimes d'un accident nucléaire. C'est Bill Hamilton qui comprend petit à petit ce qui leur arrive.

Physiquement nous sommes étendus sur le sol du bévatron. Mais mentalement nous nous trouvons ici. L'énergie du faisceau a transformé l'univers personnel de Silvester en un monde objectif. Nous sommes soumis à la logique d'un fanatique religieux. (Dick 2014a [1955]: 114)

Les personnages se retrouvent donc collectivement plongés dans l'univers subjectif fait monde, dans l'idios kosmos, de chacun de ceux qui se réveillent successivement, Arthur Silvester étant le premier à avoir repris conscience. Les mondes défilent: celui du soldat bigot où la prière permet de faire démarrer une voiture; celui d'une femme à la pudibonderie telle que les éléments du réel qui la choquent disparaissent un par un; celui d'une paranoïaque où les objets mêmes deviennent hostiles, jusqu'à la maison qui essaie de tuer les personnages; etc. On rencontre cette situation, où le narrateur et un groupe de personnages se retrouvent dans un monde qui est l'objectivation du monde subjectif d'un autre personnage, dans tous les plus grands romans de Philip K. Dick: Le Temps désarticulé (1959), Glissement de temps sur Mars, Le Dieu venu du Centaure (1964), Ubik (1966) ou Coulez mes larmes, dit le policier (1970).

\section{0}

1929. Benjamin Lee Whorf, 33 ans, se fait cryptographe et tente de déchiffrer l'écriture des anciens Maya. Recyclant la méthode étymologique mise au point par Antoine Fabre d'Olivet, il effectue une analyse componentielle de plusieurs glyphes, associant une valeur phonétique à chacun des composants arbitrairement détachés d'un caractère (Carroll 2012: 9-11, Coe 2012:135-139). 1943. Ernest Naquayouma, dont le nom signifierait Plume d'aigle, vit désormais à Chicago où il travaille dans une usine de guerre. Il exerce à l'occasion ses talents de forgeron et d'artisan traditionnel ${ }^{\mathbf{7}} .1973$. Extrait d'un article du magazine Air France Atlas:

Les Hopi ont une langue tellement sophistiquée que leurs jeunes gens s'en vont apprendre les mathématiques supérieures dans les universités américaines uniquement pour se reposer l'intellect et par façon de divertissement. Toujours d'après ce qu'on m'a expliqué la langue hopi considère le temps et l'espace comme des concepts relatifs, si bien qu'un enfant de 5 ans qui sait parler trouve les spéculations de feu Einstein tout simplement élémentaires. (Malotki 1983: 5) 
1957. Emmanuel Levinas rapproche contre toute attente Lévy-Bruhl de Heidegger en faisant tranquillement remarquer que «le temps-forme pure est inconnu des primitifs, les instants ont chacun un potentiel différent, contrairement à l'homogénéité du temps-forme. Ce potentiel tient à l'être même qui remplit l'instant, à la puissance de cet être nu " (Levinas 1957: 566). 1933. Le 2 décembre, Benjamin Lee Whorf, 36 ans, reçoit une lettre de son père, Howard, qui l'encourage à poursuivre ses recherches sur les hiéroglyphes maya en les comparant aux galets peints de la culture azilienne du sud-ouest de l'Europe: l'analogie pourrait prouver une migration transatlantique lors de l'épipaléolithique, voire même l'existence de l'Atlantide où vécut, selon la Doctrine secrète de Madame Blavatsky, la quatrième race de I'histoire humaine (Hutton et Joseph 1998: 194-195; Lee 1996: 21).

11

Manifeste testament extrait du dernier article écrit par Benjamin Lee Whorf, publié de manière posthume en 1942 dans The Theosophist à Madras:

Ma tâche est d'expliquer une idée à tous ceux qui peuvent être poussés par les évènements à jouer un rôle de premier plan dans la réorganisation globale du futur de l'humanité. C'est l'idée selon laquelle un monde nouménal - un monde d'hyperespace, de dimensions supérieures attend d'être découvert par toutes les sciences sous son aspect premier, celui du domaine des relations de structure. Cette idée est sous-entendue dans le monde des aspects préhensibles de Whitehead et dans la physique relativiste, avec son continuum à quatre dimensions et son tenseur de Riemann-Christoffel qui résume les propriétés du monde en tout point-moment. Je ne pourrais guère ajouter qu'une chose, et à cela se borne mon apport personnel, c'est qu'il existe dans le langage la prémonition d'un univers inconnu, plus vaste - un univers dont la réalité physique ne constitue que la surface ou la peau mais dans lequel nous sommes cependant et auquel nous appartenons. Car l'approche de la réalité à laquelle la connaissance moderne est en train de se livrer, par le biais des mathématiques, n'est qu'une approche à travers un cas particulier de la relation existant entre la réalité et le langage. (Whorf 2012 [1956]: 317)

L'ontologie de Philip K. Dick, c'est-à-dire l'assimilation de la multitude des mondes privés (perspectivisme) à des mondes parallèles (multivers), est toujours mise au service de la narration: c'est elle qui lui confère ses particularités. S'il a, par exemple, emprunté au Monde des Ã (1945) d'Alfred E. Van Vogt la situation initiale d'une illusion vécue comme réalité par le narrateur, il lui confère une étendue et une variabilité hors norme: ainsi le narrateur découvre soudainement qu'il n'est pas celui qu'il croyait être et que ses souvenirs sont faux (Le Temps désarticulé; Le Retour du refoulé, 1963; Souvenirs à vendre, 1965), qu'il est un androïde (L'Imposteur, 1953; La Fourmi électrique, 1968), qu'il est mort (Ubik), que sa famille est extraterrestre (Le Père truqué, 


\section{ci-contre}

fig. 7

Attribué à Mickey Bungguni, Wandijna

Figure, vers 1962, peinture sur écorce, $68,5 \times 26 \mathrm{~cm}$.

Sotheby's, Melbourne, Australie/28 juin 1999/ akg-images.

Droits réservés.
1954), que les humains sont des cadavres plus ou moins mécaniques (Glissement de temps sur Mars; La Foi de nos pères, 1966), que la ville dans laquelle il se rend n'existe pas (Le Banlieusard, 1952; Un précieux artefact, 1963), que la réalité s'effondre à intervalles réguliers (Rajustement, 1953; Le Temps désarticulé), etc.

Le schéma narratif classique où un personnage quitte le monde réel pour pénétrer dans un monde illusoire, schéma que l'on trouve, par exemple, dans L'Univers en folie de Brown ou dans L'Autre Côté du rêve (1971) d'Ursula Le Guin, est à peu près systématiquement inversé dans les récits de Dick: le réel ne précède pas l'hallucination, il lui succède. Le narrateur typique des récits de Dick prend conscience du caractère illusoire de sa réalité habituelle et découvre la vraie réalité. Mais cette découverte met à mal la distinction même entre illusion et réalité : contrairement aux récits de Brown et de Le Guin, le statut du vrai réel vacille. Si la première réalité était une illusion, pourquoi la seconde réalité, la «réalité vraie», ne serait-elle pas elle aussi une illusion? Le doute est instillé et il ne disparaîtra plus. Si un premier dévoilement a eu lieu, il peut toujours y en avoir d'autres, à l'infini. Les intrigues de Dick sont de ce fait toujours gouvernées par un art de l'escamotage de la réalité. Le réel n'est pas tué, comme chez Baudrillard; plus radicalement, il n'a jamais eu lieu.

Ainsi, dans Glissement de temps sur Mars, Jack Bohlen observe durant un épisode psychotique son directeur des ressources humaines prendre un nouvel aspect.

À travers la peau de l'homme, Jack aperçut son squelette, dont les os étaient maintenus par des filaments de cuivre. Les organes, qui s'étaient desséchés, avaient été remplacés par des composants artificiels; reins, cœur, poumons... Tout était constitué de plastique et d'acier, tout fonctionnait à l'unisson, mais sans la moindre vie réelle. La voix de l'homme provenait d'une bande magnétique, à travers un amplificateur et un haut-parleur. (Dick 2014b [1962] : 103-104)

Certes, il revient ensuite à la réalité normale et son chef reprend l'apparence d'un humain ordinaire. Mais la brèche est ouverte.

Une chose l'avait toujours inquiété depuis cet épisode psychotique avec le chef du personnel de la Corona Corporation: et si cela n'avait pas été une hallucination? Et si le soi-disant chef du personnel était vraiment tel que Jack avait pu le voir, c'est-à-dire une construction artificielle, comme ces machines éducatives? Dans ce cas, il n'y avait pas de psychose. (Ibid. : 105)

Et c'est là une caractéristique fondamentale de l'univers narratif de Philip K. Dick, au moins dans les années 1950 et 1960. II n'y a pas de réalité nouménale. II n'y a pas d'arrière-monde. Dès lors aucune certitude épistémique ne vient réconforter le lecteur. II s'attendra toujours à être surpris par une nouvelle vérité, qui ne sera jamais qu'avant-dernière. D'où l'on déduit que ce ne sont pas les personnages des romans de Dick qui sont atteints de psychose, c'est le monde lui-même qui devient psychotique. La chausse-trappe typique de l'art dickien est celle par laquelle s'achève Ubik. Le personnage principal, Joe Chip, a alors enfin compris qu'il avait été victime d'un attentat, qu'il était enfermé, en demi-vie, dans un cercueil cryogénique et que le monde 


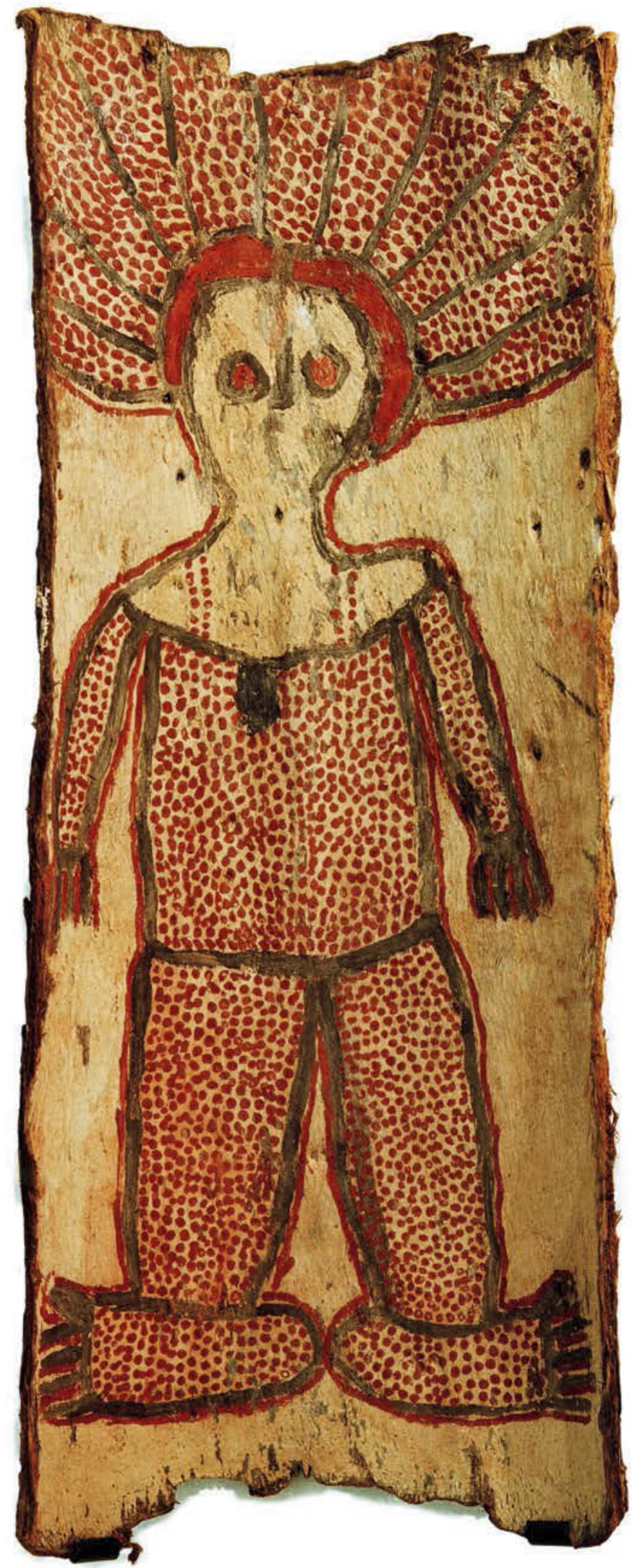


dans lequel il « vivait » était le monde subjectif de Jory Miller, un enfant mort. Il a également compris que son patron, Glen Runciter, était vivant et qu'il essayait, depuis le monde réel, d'entrer en contact avec lui. L'intrigue du roman, passablement complexe, semble donc à peu près clarifiée lorsque, dans les toutes dernières pages, Dick donne à penser qu'en fait Glen Runciter pourrait lui aussi être mort ou du moins en état de demi-vie, ultime renversement qui annihile la cohérence ontologique de tout le dispositif romanesque et libère une multiplicité de mondes définitivement dépourvue de centre ou de hiérarchie (Mucchielli 2015).

\section{3}

1908. Piotr D. Ouspenski, théosophe russe, élabore un argument déroulant les conséquences de l'existence d'une quatrième dimension: soit nous ne possédons que trois dimensions, auquel cas nous n'avons pas d'existence réelle, nous n'existons que dans l'imagination d'un être supérieur qui a vue sur nous et l'univers entier est un monde artificiel créé par son imagination; soit nous possédons nous-mêmes une quatrième dimension, auquel cas la plus grande partie de notre être vit dans la quatrième dimension et nous n'en sommes pas conscients (Rucker 1985: 92). 2437. L'homme ardent, fugguant à travers les lignes géodésiques de l'espace-temps, apprend à changer n'importe quel maintenant en réalité (Bester 2007). 1948. Certains des membres les plus éminents de la communauté hopi se réunissent à Shungopavi pour discuter d'une nouvelle interprétation de la prophétie contenue dans le mythe d'émergence du quatrième monde - le nôtre. II est dit dans ce récit que le retour de Pahaana, le Frère blanc parti à l'est à l'origine de cette humanité, serait annoncé par l'irruption soudaine de calebasses de cendre et suivi par le retour de Maasaw, divinité tutélaire qui détruirait alors ce monde pour donner naissance à un cinquième. Le problème consiste à déterminer si le Frère blanc est ou n'est pas Adolf Hitler et si la calebasse de cendre attendue n'est autre que l'explosion de la bombe atomique. 1949. Les représentants de l'Empire Hopi écrivent au président Harry Truman pour l'informer de l'imminence de la fin du monde (Geertz 1994: 140-143).

\section{4}

Philip K. Dick était un maître de l'escamotage. II construisait ses récits comme des chausse-trappes en enchâssant les mondes sans le dire et en ne dévoilant au lecteur le caractère illusoire d'un monde ou d'une subjectivité qu'a posteriori. Comme tous les grands écrivains de science-fiction, il aimait s'attacher au sens littéral des métaphores pour mieux les développer (assez typiquement, lorsqu'un écrivain de science-fiction reprend l'expression «Dieu est mort», c'est pour se demander comment affréter un supertanker qui pourra remorquer son cadavre), ignorant volontairement les guillemets épistémologiques qui permettent d'ordinaire de comprendre qu'un manteau de neige n'a pas de bras. Et il recycla dans ses romans les visions les plus déconcertantes et les plus épouvantables des schizophrènes en escamotant sciemment la distanciation qu'ils conservent toujours vis-à-vis de leurs délires, cette forme de réflexivité connue des psychiatres sous les noms de “concrétude fantôme» et de «familiarité inquiétante et muette» (Sass 2010). Les mondes enchâssés de Philip K. Dick, hallucinés ou délirants, sont presque 
toujours présentés comme des mondes ordinaires, sans guillemets, c'està-dire sans la méta-représentation spécifiant que "le monde X" est le délire d'un psychotique » ou que «"le monde $Y$ " est l'hallucination d'un drogué ". Les mondes $X$ et $Y$ sont donnés tels quels, escamotés, truqués, et ce pour le plus grand plaisir des lecteurs: ce n'est qu'à cette condition que l'on peut partager l'illusion d'une pensée radicalement autre.

J'ai essayé de montrer comment Benjamin Lee Whorf, pour se donner l'illusion de découvrir lui aussi une pensée autre, mit en place un dispositif intellectuel semblable à celui des écrivains de science-fiction en général et à celui de Philip K. Dick en particulier ${ }^{8}$. II commença ainsi en branchant le grand commutateur dichotomique. Il devait nécessairement exister d'un côté la «pensée du même», la nôtre, et d'un autre côté la «pensée autre», la leur, celle des «Hopi». Avec le commutateur on pouvait imaginer deux mondes de personnages conceptuels. Le premier serait évidemment monolithique. Il comprendrait la logique, la science, l'anthropologie, la pensée de l'Occident dans une version "génération perdue". Le second serait apparemment plus variable puisque autre. Mais cette variété exotique se limiterait bien vite - effet du commutateur dichotomique - à une pensée pauvre, bientôt réduite à une inversion systématisée de quelques aspects de la «pensée occidentale», la pensée de référence.

Le processus de construction de la pensée autre se poursuivait par un escamotage qui consista à faire passer pour la pensée des autres une variante de sa propre tradition intellectuelle. Whorf retrouva ainsi une interprétation cosmologique de la théorie de la relativité dans la langue et la pensée des Hopi. C'était là ce qu'on pourrait appeler une anthropologie ventriloque, pratique de marionnettes et de marionnettistes, où la pensée du dehors se révélait cacher une pensée du dedans, où les philosophes morts s'exprimaient à travers les Amérindiens vivants, à leur insu.

Pratique aux effets délétères, s'il en est; le principal étant un désintérêt total vis-à-vis des capacités réflexives propres aux Hopi. En escamotant la différence entre savoir et savoir sur le savoir, en fusionnant des schèmes réputés inhérents à la langue des Hopi avec ses obsessions métaphysiques, Benjamin Lee Whorf s'interdisait de prendre en compte les modes de pensée, traditionnels ou non, des Hopi eux-mêmes, leur déniant dans les faits toute réflexivité propre et autonome dans le seul but d'édifier une position originale dans le champ de bataille des philosophies occidentales.

Laboratoire d'anthropologie sociale pierredeleage@gmail.com
8. Sur la postérité de Benjamin Lee Whorf dans la littérature de sciencefiction, voir: Yaguello 2006: 126-133; Landragin 2018. 
Manhattan Transfer. Benjamin Lee Whorf diffracté par Philip K. Dick

Par Pierre Déléage

\section{Bergson, Henri}

2009 [1922] Durée et

simultanéité. Paris, PUF.

\section{Bester, Alfred}

2012 [1956] Terminus les étoiles, trad. de l'anglais par Patrick Marcel. Paris, Folio SF.

\section{Briggs, Kevin C.} et D. Scott Apel

1992 «Entretien avec Philip K. Dick» (1977), in Hélène Collon (éd.), Regards sur Philip K. Dick: le kalédickoscope. Anthologie de témoignages et de textes critiques. Amiens, Encrage: : 79-101.

\section{Carroll, John B.}

2012 [1956] "Introduction», in Benjamin Lee Whorf, Language, Thought and Reality. Cambridge, MIT Press.

\section{Coe, Michael D.}

2012 [1992] Breaking the Maya Code. Londres, Thames \& Hudson.

\section{Darnell, Regna}

2001 Invisible Genealogies:

A History of Americanist

Anthropology. Lincoln, University of Nebraska Press.

\section{Deleuze, Gilles}

1988 Le Pli : Leibniz et le baroque. Paris, Minuit.

\section{Dick, Philip K.}

1996a «Letter to James Blish. June 7, 1964 ", in The Selected Letters of Philip K. Dick, t. I, 1938-1971. Novato, Underwood Books : 77-78.

1996b "Letter to Bruce Gillepsie. June 8, 1969 ", in Ibid. : 263-264.

1996c «Drugs, hallucinations and the Quest for Reality » (1964), in Lawrence Sutin (éd.), The Shifting Realities of Philip K. Dick.

New York, Vintage Books: 167-174

1996d «Schizophrenia and the Book of Changes " (1965), in Ibid. 175-182.

1998 "Le hibou ébloui » (1981), in Nouvelles, t. II, 1963-1981, éd. par Hélène Collon. Paris, Denoël : 1372-1377.
$2014 a$ [1955] L'CEil dans le ciel, trad. de l'anglais par Gérard Klein. Paris, J'ai lu.

2014b [1962] Glissement de temps sur Mars, trad. de l'anglais par Henry-Luc Planchat. Paris, J'ai lu.

\section{Einstein, Albert}

1939 "Letter to Franklin D. Roosevelt, August 2, 1939 ", Wikisource.

\section{Geertz, Armin W}

1994 The Invention of Prophecy. Berkeley, University of California Press.

\section{Heynick, Frank}

1983 «From Einstein to Whorf: Space, Time, Matter, and Reference Frames in Physical and Linguistic Relativity ", Semiotica 45: 35-64.

\section{Hutton, Christopher M.} et Joseph, John E.

1998 «Back to Blavatsky: The Impact of Theosophy on Modern Linguistics ", Language and Communication 18: 181-204.

\section{Landragin, Frédéric}

2018 Comment parler à un alien? Langage et linguistique dans la science-fiction. Saint Mammès Le Bélial.

\section{Lee, Penny}

1991 «Whorf's Hopi Tensors: Subtle Articulators in the Language/ Thought Nexus? ", Cognitive Linguistics 2 (2): 123-148.

\section{Lee, Penny}

1996 The Whorf Theory Complex. Philadelphia, John Benjamins Publishing Company.

\section{Lehman, Serge}

2001 "La physique des métaphores », Europe 870: 32-50.

\section{Levinas, Emmanuel}

1957 «Lévy-Bruhl et la philosophie contemporaine", Revue philosophique de la France et de l'Etranger 147: 556-569.

\section{Livingstone, Robert \\ 1963 "Perception and \\ Commitment ", Bulletin of the Atomic Scientists 19 (2) : 14-18.}

\section{Malotki, Ekkehart}

1983 Hopi Time: A Linguistic Analysis of the Temporal Concepts in the Hopi Language. Berlin, Mouton Publishers.

May, Rollo, Angel, Ernest et Ellenberger, Henri F. (dir.)

1958 Existence: A New Dimension in Psychiatry and Psychology. New York, Basic Books.

\section{Mucchielli, Jacques}

2015 "La réalité avant-dernière ", in Richard Comballot (dir.), Philip K. Dick: Simulacres et illusions. Chambéry, ActuSF : 237-248.

\section{Platt, Charles}

2015 "Entretien avec Philip K. Dick» (1979), in Ibid. : 271-286.

\section{Rollins, Peter C.}

1971 "Benjamin Lee Whorf: Transcendental Linguist ", Journal of Popular Culture 5: 660-672.

1980 Lost Generation: Theories of Mind, Language, and Religion Bowling Green, Popular Culture Association.

\section{Rucker, Rudy}

1985 La Quatrième Dimension, trad. de l'anglais par Christian Jeanmougin. Paris, Seuil.

\section{Sass, Louis A.}

2010 [1994] Les Paradoxes du délire: Schreber, Wittgenstein et l'esprit schizophrénique, trad. de l'anglais par Pierre-Henri Castel. Paris, Ithaque.

\section{Schuchardt, Charlotte}

1950 «Alfred Habdank Skarbek Korzybski: A Biographical Sketch», General Semantics Bulletin 3: 33-40.

\section{Whorf, Benjamin Lee}

1946 "The Hopi Language, Toreva Dialect ", in Harry Hoijer (dir.), Linguistic Structures of Native America. New York, Viking Fund Publications in Anthropology 6 : 158-183.
2012 [1956] Language, Thought and Reality. Cambridge, MIT Press.

\section{Yaguello, Marina}

2006 [1984] Les Langues imaginaires: mythes, utopies, fantasmes, chimères et fictions linguistiques. Paris, Seuil. 


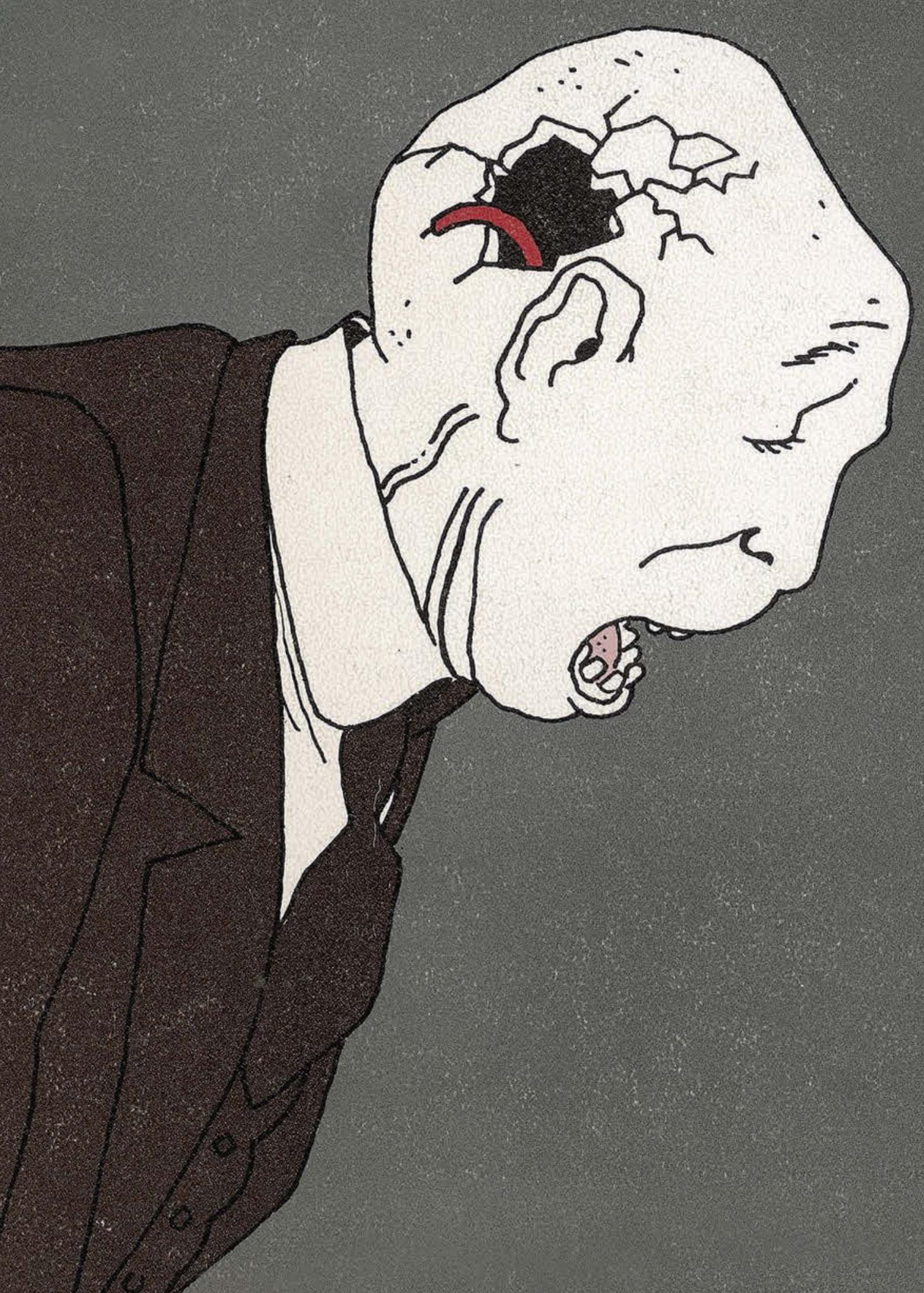

